\title{
Modified-release dipyridamole combined with aspirin for secondary stroke prevention
}

\author{
Hans-Christoph Diener \\ University Essen, Department \\ of Neurology, Hufelandstr. \\ 55, 45147 Essen, Germany \\ Tel.: +492017232460; \\ Fax: +49201723 5901; \\ E-mail: h.diener@ \\ uni-essen.de
}

Patients suffering from transient ischemic attack (TIA) or ischemic stroke have a high risk of suffering a first or recurrent stroke, with an annual risk of between 5 and $15 \%$. The European Stroke Prevention Study (ESPS) 2 was a randomized, double-blind, placebocontrolled trial involving 6602 patients with TIA or stroke comparing aspirin alone $(50 \mathrm{mg}$ daily), modified-release (MR) dipyridamole alone (200 mg twice daily), aspirin plus dipyridamole, and placebo. The 2-year relative risk reduction of stroke in the aspirin plus MR-dipyridamole group (37.0\%) was significantly higher than in either the aspirin group $(18.1 \%)$ or the MR-dipyridamole group (16.3\%). The results of the comparison between aspirin plus MR-dipyridamole versus placebo confirmed the findings of ESPS1. The results of ESPS2 were at odds with all prior trials with dipyridamole alone or in association with aspirin, but it was also the only trial sufficiently powered to show a significant difference. For this reason, a meta-analysis was performed based on a systematic review of individual patient data from randomized controlled trials involving dipyridamole in patients with prior ischemic stroke or TIA. Recurrent stroke was reduced by dipyridamole compared with placebo, and by combined aspirin and dipyridamole versus aspirin alone, dipyridamole alone or placebo. In two post hoc analyses the efficacy of aspirin plus extended-release dipyridamole was compared with aspirin alone for the prevention of recurrent stroke among high-risk groups. Stroke models from the Framingham Study and the Stroke Prognostic Instrument II were applied to subjects in ESPS2 to assign patients to risk groups. Compared with aspirin alone, aspirin plus MR-dipyridamole demonstrated a more pronounced efficacy in reducing the risk for stroke and vascular events among patients younger than 70 years of age, with hypertension, or prior stroke; current smokers; and those with any prior cardiovascular disease. Another model (Essen risk score) showed that patients with a high risk of recurrent stroke show a much greater benefit from aspirin plus MR-dipyridamole compared with aspirin monotherapy than patients with a low risk.

Annually, 15 million people worldwide suffer a stroke, of whom 5 million die and 5 million are permanently disabled [1]. The age-related incidence of stroke is declining in many developed countries, largely as a result of the control of risk factors such as high blood pressure and smoking. The absolute number of strokes continue to increase because of the aging population. For example, stroke is the biggest single cause of disability in the UK. People who have an ischemic stroke or transient ischemic attack (TIA) are at high risk of a second stroke [2], although this risk ranges from approximately 5\% to around $20 \%$ per year $[3,4]$. The recurrence risk is highest in the first few days after a TIA or stroke and declines with time. Such second strokes often cause substantial disability and greatly reduce quality of life.

Evidence-based improvements to the prevention of second strokes by modifying risk factors and by pharmacologic means can greatly reduce the burden of disability and the costs of stroke. The overall value of antiplatelet therapy for the prevention of second strokes has been established, as has the value of aspirin as a contributor $[5,6]$. The best antithrombotic regimens for individual patients remain unclear, but the evidence needed for more effective prescribing is steadily increasing. Current evidence suggests that the combination of modified-release (MR) dipyridamole and low-dose aspirin in a single MR-dipyridamoleaspirin formulation $\quad\left(\right.$ Aggrenox ${ }^{\circledR} /$ Asasantin $^{\circledR}$ Retard, Boehringer Ingelheim, Germany) should be a highly considered option.

\section{Pharmacology}

The antithrombotic activity of MR-dipyridamoleaspirin appears to result from additive pharmacologic effects of aspirin and MR-dipyridamole. Aspirin alters prostaglandin metabolism and irreversibly inhibits cyclooxygenase-1, suppressing 
thromboxane $\mathrm{A}_{2}$ synthesis, and thus platelet function $[7,8]$. It is an effective antithrombotic agent even at low doses (50-100 mg/day and possibly lower) [9]. Dipyridamole is a weak inhibitor of platelet aggregation, and may produce its antithrombotic effects through other mechanisms mainly related to the vessel wall (including effects on the endothelium, antioxidant effects and antiinflammatory effects [10]). In clinical studies the bleeding rate with the combination is not significantly higher than that with aspirin alone (unlike other combinations of antiplatelet agents, e.g., clopidogrel plus aspirin [11]). This supports the view that the improved efficacy of the combination, compared with aspirin alone, results from biologic actions of dipyridamole other than antiplatelet activity, probably its activity on the endothelium.

Problems with reduced absorption of dipyridamole in people with high gastric $\mathrm{pH}$ and potential compliance problems with the earlier regimen of three daily doses led to the development of a formulation of MR-dipyridamole with aspirin, which has a tartaric acid core. This formulation keeps plasma levels of dipyridamole within the therapeutic range for longer, necessitating only two daily doses. The tartaric acid core makes the release and absorption of dipyridamole almost independent of the gastric $\mathrm{pH}$, thus avoiding the problem of reduced absorption where gastric $\mathrm{pH}$ is raised (in older people and others taking antacids or $\mathrm{H}_{2}$ blockers). The resulting decrease in fluctuations of plasma dipyridamole levels may improve efficacy by providing a more sustained protective effect on the vessel wall (endothelium). The slower increase in the plasma concentration of dipyridamole also reduces any possible risk for those with coronary heart disease.

\section{Efficacy}

Some earlier trials did not show superiority of the combination of aspirin with dipyridamole over aspirin alone, but these studies did not use the newer formulation and may not have been adequately powered to detect a superior effect of the combination. The Accidents Ischémiques Cérébraux Liés à l'Athérosclérose (AICLA) double-blind randomized trial compared aspirin alone ( $1 \mathrm{~g} /$ day) and dipyridamole $(225 \mathrm{mg}$ /day) plus aspirin $(1 \mathrm{~g} /$ day) with placebo, for 3 years, in 604 patients who had experienced TIAs or ischemic strokes [12]. Rates of fatal and nonfatal strokes showed a strong trend between aspirin plus dipyridamole and placebo $(\mathrm{p}<0.06)$, and a significant difference between aspirin monotherapy and placebo $(\mathrm{p}<0.05)$ but no significant difference between aspirin plus dipyridamole and aspirin alone. The Persantine Aspirin Trial randomized 890 people with recent carotid territory TIAs to either aspirin $(325 \mathrm{mg}$ ) plus placebo or aspirin $(325 \mathrm{mg}$ ) plus dipyridamole $(75 \mathrm{mg}$ ) four-times daily, and followed them for 1-5 years [13]. Overall end point rates (stroke or death) for the aspirin-only and aspirin plus dipyridamole groups were identical. Similarly, the Antiplatelet Trialists' Collaboration analysis of antiplatelet treatment trials did not find a significant difference between combination treatment and aspirin alone [14]. The European Stroke Prevention Study (ESPS) 1 trial compared coprescription of dipyridamole (75 mg) and aspirin (330 mg) three-times daily with placebo in 2500 patients with a previous stroke or TIA [15]. This treatment resulted in a $33.5 \%$ reduction in the incidence of the combined end point (deaths from all causes or stroke) and a 38\% reduction in stroke incidence compared with placebo, a much greater reduction than would be expected with aspirin alone.

ESPS2 is the only trial of the combination in which the MR formulation of dipyridamole has been used [16]. In ESPS2, 6602 patients with stroke or TIA were randomly assigned to four treatment arms: placebo; aspirin alone $(25 \mathrm{mg}$ twice daily); MR-dipyridamole alone (200 mg twice daily); or MR-dipyridamole (200 mg twice daily) plus aspirin (25 mg twice daily).

Both aspirin and dipyridamole, individually, were equally effective for the secondary prevention of ischemic stroke and TIA. When given as monotherapy, each agent in the combination was associated with a significant reduction in stroke risk compared with placebo: $18 \%$ $(\mathrm{p}=0.013)$ for aspirin, and 16\% ( $\mathrm{p}=0.039)$ for dipyridamole (Figure $1 \& 2$ ). The combination was significantly more effective than either drug alone at reducing the outcome of stroke and marginally more effective at reducing stroke and/or death; the $37 \%$ reduction $(\mathrm{p}=0.001)$ in stroke risk over 2 years, compared with placebo, was similar to the risk reduction observed in the ESPS 1 trial. Neither the combined treatment nor the single drugs had any significant effect on death rate alone. Individually, both aspirin and dipyridamole significantly reduced the incidences of TIA (risk reductions of 22 and 18\%, respectively), and the combination reduced the risk by $36 \%$ compared with placebo $(\mathrm{p}<0.001)$. 
Figure 1. Pairwise comparisons of the relative risk reductions (stroke) with different treatments in the ESPS2 trial.

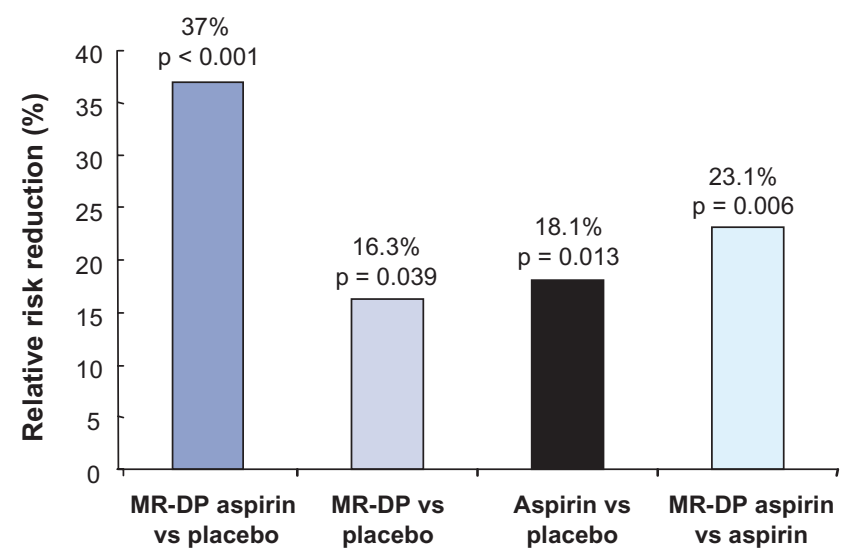

Based on data from [16]. MR-DP: Modified-release dipyridamole.

A meta-analysis based on a systematic review of all the available individual patient data from

Figure 2. Stroke-free survival of patients with prior stroke or TIA treated with aspirin, dipyridamole, MR-dipyridamoleaspirin or placebo in the ESPS-2 trial.

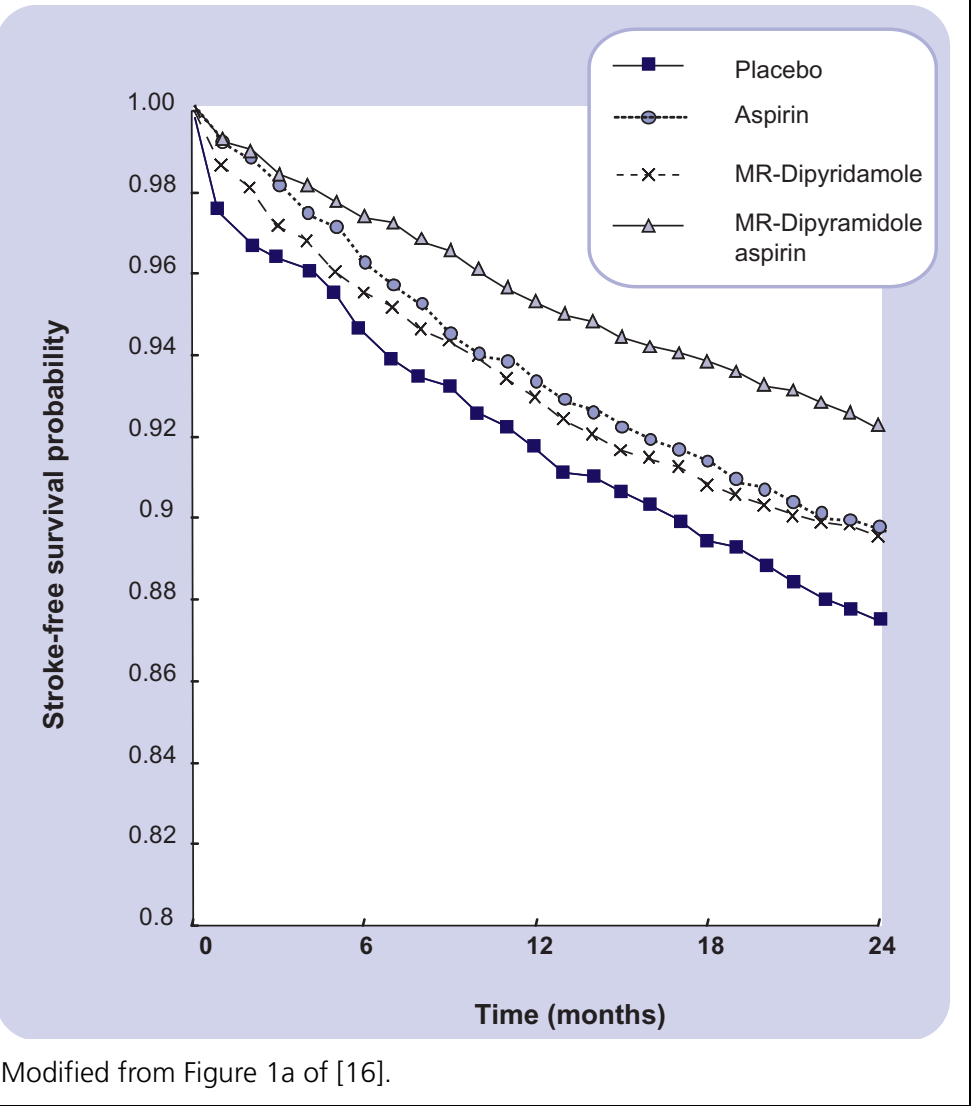

randomized controlled trials involving dipyridamole in patients with prior ischemic stroke or TIA confirms the additive effects of aspirin and dipyridamole [17]. Dipyridamole was compared with placebo, and combined aspirin and dipyridamole with the monotherapies and with placebo. Individual patient data were available for 11,036 patients. Recurrent stroke was reduced by dipyridamole compared with placebo (odds ratio $[\mathrm{OR}] \quad 0.82,95 \%$ confidence interval [95\% CI] 0.68-1.00), and by combined aspirin and dipyridamole compared with aspirin alone (OR 0.78, 95\% CI 0.65-0.93), dipyridamole alone (OR $0.74,95 \%$ CI $0.60-0.90$ ) or placebo (OR $0.61,95 \%$ CI 0.51-0.71). The combination of aspirin and dipyridamole also significantly reduced the composite outcome of nonfatal stroke, nonfatal myocardial infarction (MI) and vascular death as compared with aspirin alone (OR 0.84, 95\% CI 0.72-0.97), dipyridamole alone (OR 0.76 , 95\% CI $0.64-0.90$ ) or placebo (OR 0.66, 95\% CI 0.57-0.75).

A post hoc analysis of data from ESPS2 indicates that the efficacy advantage of combined MR-dipyridamole-aspirin over aspirin alone is greater in higher-risk patients [18]. Annual rates of strokes and vascular events for the MR-dipyridamole-aspirin group $(\mathrm{n}=1650)$ and the aspirin-only group $(\mathrm{n}=1649)$ were stratified according to both risk subgroups and individual risk factors. Stroke models from the Framingham Study and the Stroke Prognostic Instrument II (SPI-2) were used to divide ESPS2 patients into risk groups. Compared with aspirin alone, MR-dipyridamole-aspirin produced the greatest reduction in the risk of stroke and vascular events in patients younger than 70 years of age, those with hypertension, prior stroke or TIA, current smokers, and those with any prior cardiovascular disease. Relative hazard reductions were largest with the MR-dipyridamoleaspirin combination and were most notable for the high-risk group, based on the Framingham model; and for the moderate-risk subgroup based on the SPI-2 model. This apparent inconsistency with the two models may have been because the numbers of patients allocated to the highest risk group, based on SPI-2, were small.

In another post hoc analysis the authors applied a risk score developed from the Clopidogrel versus Aspirin in Patients at Risk of Ischemic Events (CAPRIE) trial (Table 1) to the population of ESPS2. The category 'other cardiovascular' included ischemic heart disease, cardiac failure and ventricular arrhythmia. As 
Table 1. Essen risk score based on a subgroup analysis of stroke patients in the CAPRIE trial. A total score of 3 or more reflects a high recurrence risk for stroke ( $\geq 4 \% /$ year).

\begin{tabular}{lr} 
Risk factor & Points \\
\hline$<65$ years & 0 \\
$65-75$ years & 1 \\
$>75$ years & 2 \\
Hypertension & 1 \\
Diabetes & 1 \\
Previous MI & 1 \\
Other cardiovascular disease (except MI and atrial fibrillation) & 1 \\
Peripheral arterial disease & 1 \\
Smoker & 1 \\
\hline Additional TIA or ischemic stroke in addition to qualifying event & 1 \\
\hline
\end{tabular}

CAPRIE: Clopidogrel versus Aspirin in Patients at Risk of Ischemic Events; MI: Myocardial infarction; TIA: Transient ischemic attack.

can be seen in Figure 3, patients with a low risk score of $0-2$ show no difference between aspirin and MR-dipyridamole-aspirin. In patients with a higher risk score, the combination is clearly superior to aspirin monotherapy. This result shows that stratification of patients according to risk of recurrent stroke will lead to different treatment regimens.

\section{Tolerability \& safety}

In the ESPS2 trial, headache was the most common adverse event and occurred in $37-38 \%$ of dipyridamole-treated patients compared with $32-33 \%$ of those receiving aspirin alone or placebo $(\mathrm{p}<0.001)$ [16]. In patients who received dipyridamole (monotherapy or combined with aspirin), headache was the most common reason for discontinuing drug intake: $8 \%$ of patients in the dipyridamole groups and $2 \%$ in the aspirinonly or placebo groups discontinued because of headache.

The incidence and severity of headaches decreased rapidly within the first few days in a study in which volunteers took dipyridamole, indicating that most patients quickly develop tolerance [19]. In volunteers $(\mathrm{n}=36)$ who took combination treatment for two periods of 5 days separated by 3 days' washout, headaches (mainly mild) affected $67 \%$ on the first day of treatment but only $31 \%$ on the third day and only $3 \%$ by the fourth day of the second treatment period.

A pilot study of initial dose titration suggested that titration with an initially lower dose of dipyridamole may reduce headache and increase compliance, although the differences seen were not significant [20]. Patients were randomized to standard aspirin and dipyridamole treatment twice daily for 2 weeks or to aspirin only in the morning, and aspirin and dipyridamole in the evening for 5 days, followed by 9 days of standard aspirin and dipyridamole treatment. Moderate-to-severe headache was reported by $28 \%$ in the standard-treatment group and $25 \%$ in the titration group, but the cumulative number of days with headache was more than twice as high in the standard group compared with the titration group.

Patients starting MR-dipyridamole-aspirin therapy should be prepared for the possibility of headache and asked to persevere with treatment until the headache goes. They should not discontinue treatment for any prolonged period. In patients who are either considered prone to headache (history of migraine) or who report moderate-to-severe headache after a first dose, it may be helpful to start treatment with one capsule at night for the first 7-14 days, followed by escalation to the recommended dose. The initial titration dose should not be considered effective for stroke prevention.

In the ESPS2 study gastrointestinal events, including diarrhea, were also common, particularly in the patients treated with dipyridamole and aspirin. The percentages of patients who discontinued because of gastrointestinal events were $4 \%$ in the placebo and aspirin-only groups, $6 \%$ in the dipyridamole-only group and $7 \%$ in the dipyridamole plus aspirin group.

All-site bleeding episodes and gastrointestinal bleeds were significantly more frequent and more often moderate or severe/fatal in patients receiving aspirin compared with those who were not. However, bleeding episodes were not significantly more frequent in patients receiving combination treatment than in those receiving aspirin alone. Bleeding at any site occurred in $8.7 \%$ of those receiving the aspirin-dipyridamole combination, $8.2 \%$ of those receiving aspirin alone, $4.7 \%$ of those on dipyridamole alone and $4.5 \%$ of the placebo group. Similarly, there was no significant difference between rates of severe or fatal bleeding in the MR-dipyridamole-aspirin group compared with the aspirin monotherapy group (Figure 4).

The belief that dipyridamole is harmful in cardiac patients (patients with MI or coronary heart disease) is not justified. Post hoc analysis of cardiac events in those with coronary heart disease or MI at entry to the ESPS2 study showed that dipyridamole neither decreased nor increased the 
Figure 3. Annual event rates (\%) from the ESPS2 trial based on the Essen risk score (see table 1) for aspirin monotherapy (dark columns) and the MR-DP-aspirin combination therapy.

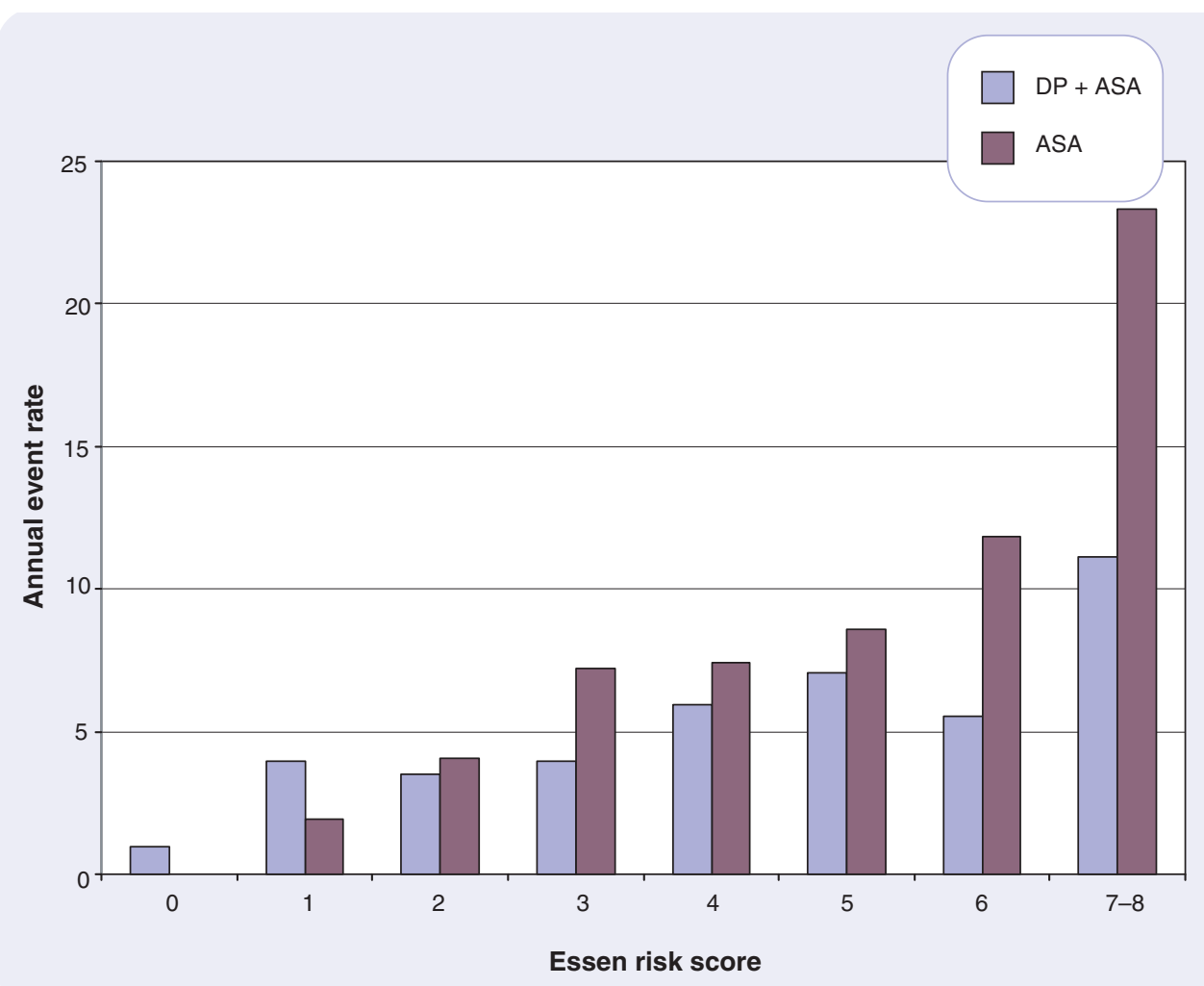

number of cardiac events (angina pectoris, MI or death from all causes) [21]. The Prevention Regime For Effectively avoiding Second Strokes (PRoFESS) trial (discussed below) will assess whether MR-dipyridamole-aspirin is beneficial in such patients.

\section{Comparison with other antithrombotic options}

The other options that are currently available for antithrombotic therapy are aspirin, dipyridamole or clopidogrel, as monotherapies, or the combination of clopidogrel with aspirin. Direct comparison of MR-dipyridamole-aspirin with aspirin alone in ESPS2 indicates the superiority of MR-dipyridamole-aspirin. MRdipyridamole-aspirin combination treatment was also shown to be superior to dipyridamole monotherapy in ESPS2.

Clopidogrel and MR-dipyridamole-aspirin have never previously been directly compared. In the CAPRIE study in patients with atherosclerotic vascular disease (ischemic stroke, nonfatal MI or peripheral arterial disease) clopidogrel

$75 \mathrm{mg}$ once daily compared favorably with aspirin $75 \mathrm{mg}$ [22]. Clopidogrel produced an $8.7 \%$ relative reduction in the risk of ischemic stroke compared with aspirin (95\% CI 0.3-16.5). Clopidogrel was also slightly more effective than aspirin in the subgroup of patients with ischemic stroke (relative risk reduction $7.6 \%, 95 \% \mathrm{CI}$ $-0.8-15.3)$ but this study was not sufficiently statistically powered to detect differences within the subgroups.

The need for a direct comparison of the MRdipyridamole-aspirin combination with clopidogrel is being addressed in the PRoFESS trial, which is currently underway. PRoFESS is comparing MR-dipyridamole-aspirin with clopidogrel (Table 2). PRoFESS has a two by two factorial design with a separate randomization to the angiotensin receptor blocker telmisartan or placebo (in addition to standard blood-pressure lowering therapy). The study aims to recruit 18,500 patients aged over 50 years who have had a recent ischemic stroke or TIA. The primary outcome is time to recurrent stroke, while the three secondary outcomes are: 
Table 2. PRoFESS trial study design.

\begin{tabular}{llr}
\hline Agent & Plus & n \\
\hline MR-dipyridamole-aspirin & Clopidogrel placebo and telmisartan§ & 4625 \\
& Clopidogrel placebo and telmisartan placebo & 4625 \\
\hline Clopidogrel $^{\ddagger}$ & MR-dipyridamole-aspirin placebo and telmisartan§ & 4625 \\
& MR-dipyridamole-aspirin placebo and telmisartan placebo & 4625 \\
\hline
\end{tabular}

*MR-dipyridamole-aspirin: $200 \mathrm{mg} / 25 \mathrm{mg}$ twice daily.

${ }^{\mp}$ Clopidogrel: $75 \mathrm{mg}$ once daily.

§Telmisartan: $80 \mathrm{mg}$ once daily.

MR: Modified release; PRoFESS: Prevention Regime For Effectively avoiding Second Strokes.

- A composite vascular events outcome (time to first stroke, MI or vascular death);

- Composite of stroke, MI, vascular death or chronic heart failure;

- New diagnosis of diabetes.

The trial will also consider several tertiary outcomes. Recruitment is now well underway.

The combination of clopidogrel and aspirin was studied in the Management of ATherothrombosis with Clopidogrel in High-risk patients (MATCH) trial in patients with recent ischemic stroke or TIA and at least one additional vascular risk factor. This combination did not significantly reduce the risk of ischemic events compared with clopidogrel alone, and patients taking the combination had significantly more bleeding episodes [11]. Combination therapy with clopidogrel and aspirin cannot therefore be recommended in patients with TIA or stroke and at high risk of

Figure 4. Incidences of severe or fatal bleeding of patients with prior stroke or TIA treated with aspirin, dipyridamole, MR-dipyridamole-aspirin or placebo in the ESPS2 trial.

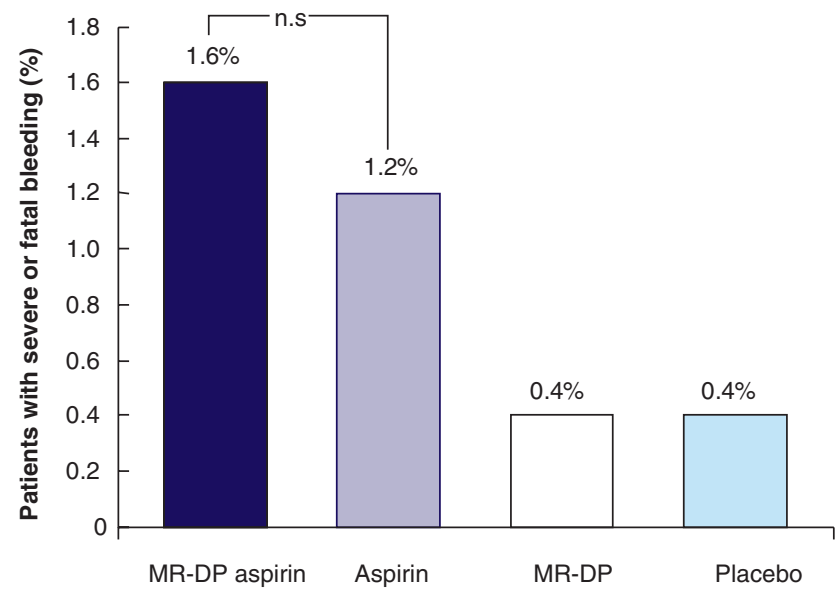

MR-DP: Modified-release dipyridamole; n.s: Not significant recurrence. After the results of the MATCH trial became known, the planned comparator in the PRoFESS trial was changed from the combination of clopidogrel and aspirin to clopidogrel alone.

\section{Treatment guidelines \& evidence-based prescribing}

Most current guidelines (such as those from the European Stroke Initiative 2003 [23], The American College of Chest Physicians [ACCP] 2004 [24] and The Royal College of Physicians 2004 [25]) include the combination of aspirin and MR-dipyridamole as a first-line treatment option for the secondary prevention of stroke alongside aspirin and clopidogrel monotherapy. However, in light of the current evidence, some of the more recently issued recommendations suggest that MR-dipyridamole combined with aspirin may be preferable to aspirin monotherapy and possibly to clopidogrel monotherapy (e.g., ACCP 2004 [26]; National Institute for Health and Clinical Excellence 2005 [26]). One of the points in favor of the combination is its positive cost-benefit ratio for stroke prevention (at least for the first 2 years after stroke) [27].

The German Neurological Society will give the following recommendations in the 2005 guidelines on secondary stroke prevention. Patients with a low recurrence risk of less than $4 \% /$ year calculated by the Essen risk score (Table 1) should be treated with aspirin monotherapy $(100 \mathrm{mg} /$ day $)$. Patients with a high recurrence risk of more than $4 \% /$ year should receive the MR-dipyridamole-aspirin combination therapy. Patients with a high recurrence risk and peripheral arterial disease should receive clopidogrel monotherapy. The group of patients with peripheral arterial disease had the highest benefit from clopidogrel in the CAPRIE trial [22]. Patients who are intolerant to aspirin or have contraindications should receive clopidogrel. 


\section{Future perspective}

Prevention of recurrent stroke with antiplatelet medication has reached a ceiling. Drugs that are more effective than aspirin will lead to very small or nonexistent increases in benefit (avoidance of second strokes) but will lead to more bleeding complications. This has been shown in the MATCH trial for the combination of clopidogrel plus aspirin versus clopidogrel monotherapy [11] Glycoprotein IIb/IIIa antagonists are more potent antiplatelet drugs than aspirin, but have also failed in the secondary prevention of stroke due to the increased rate of bleeding complications [28]. The future of stroke prevention, therefore, lies is in the optimal treatment of vascular risk factors and in drugs that offer protection to the endothelium.

\section{Acknowledgements}

Dan Cotton and Jon Blatchford performed additional statistical analyses on the ESPS2 database. Margaret Gray provided support with the first draft of the manuscript.

Prof. Dr. HC Diener received research grants or was a consultant or speaker for the following companies: AstraZeneca, GlaxoSmithKline, MSD, Pfizer, Lilly, Boehringer Ingelheim, Janssen-Cilag, Novartis, SanofiAventis, Servier and Solvay.

\section{Executive summary}

\section{Efficacy}

- The European Stroke Prevention Study (ESPS)2, the only trial to use the current modified-release (MR) dipyridamole-aspirin formulation, found the combination to be superior to either drug alone in preventing strokes in people with recent ischemic stroke or transient ischemic attack (TIA).

- The combination reduced stroke risk by $37 \%$ compared with placebo $(p=0.001)$ over 2 years.

- Post hoc analysis of cardiac events in those patients with coronary heart disease or myocardial infarction at entry to ESPS2 showed that dipyridamole is not effective in preventing cardiac events but neither is it harmful in such patients.

\section{Tolerability \& safety}

- In the ESPS2 trial, bleeding episodes were not significantly more frequent with combination therapy than with aspirin alone.

- Headaches were the most frequent reason for discontinuation in patients taking dipyridamole in ESPS2. Their incidence and severity decreases rapidly within the first few days if patients persist with treatment.

\section{Comparison with other antithrombotic options}

- MR-dipyridamole-aspirin and clopidogrel are being directly compared in the ongoing Prevention Regime For Effectively avoiding Second Strokes (PRoFESS) trial.

\section{Treatment guidelines \& evidence-based prescribing}

- Based on current evidence, most guidelines include MR-dipyridamole-aspirin as a first-line option for secondary prevention after ischemic stroke or TIA, and some recent versions suggest it may be preferable to the other options.

\section{Bibliography}

1. Mackay J, Mensah GA: The Atlas of Heart Disease and Stroke. World Health Organization, Geneva, Switzerland, 50 (2004).

2. Albers GW: Choice of end points in antiplatelet trials. Which outcomes are most relevant to stroke patients? Neurology 54 , 1022-1028 (2000).

3. Dennis M, Bamford J, Sandercock P, Warlow C: The prognosis of transient ischemic attacks in the community. The Oxfordshire Community Stroke project. Stroke 21, 848-853 (1990).

4. Petty GW, Brown RD Jr, Whisnant JP, Sicks JD, O'Fallon WM, Wiebers DO: Ischemic stroke subtypes: a population-based study of functional outcome, survival and recurrence. Stroke 31, 1062-1068 (2000).

5. Antithrombotic Trialists' Collaboration: Collaborative overview of randomized trials of antiplatelet therapy. Prevention of death, myocardial infarction, and stroke by prolonged antiplatelet therapy in various categories of patients. Br. Med. J. 308(6921), 81-106 (1994).

6. Antithrombotic Trialists' Collaboration: Collaborative meta-analysis of randomized trials of antiplatelet therapy for prevention of death, myocardial infarction, and stroke in high-risk patients. Br. Med. J. 324, 71-86 (2002).
7. Roth GJ, Stanford N, Majerus PW: Acetylation of prostaglandin synthase by aspirin. Proc. Natl Acad. Sci. USA 72(8), 3073-3076 (1975).

8. Smith JB, Willis AL: Aspirin selectively inhibits prostaglandin production in human platelets. Nature New Biol. 231(25), 235237 (1971).

9. Patrono C, Coller B, Dalen JE et al.: Platelet-active drugs. The relationships among dose, effectiveness, and side effects. Chest 114(Suppl.), S470-S488 (1998).

10. Eisert WG: Dipyridamole. In: Platelets. Michelsen A (Ed.), Academic Press, NY, USA, 803-815 (2002). 
11. Diener HC, Bogousslavsky J, Brass LM et al: Aspirin and clopidogrel compared with clopidogrel alone after recent ischaemic stroke or transient ischaemic attack in highrisk patients (MATCH): randomized, double-blind, placebo-controlled trial. Lancet 364(9431), 331-337 (2004).

12. Bousser MG, Eschwege E, Haguenau M et al: 'AICLA' controlled trial of aspirin and dipyridamole in the secondary prevention of athero-thrombotic cerebral ischemia. Stroke 14(1), 5-14 (1983).

13. American-Canadian Co-operative Study Group: Persantine Aspirin Trial in cerebral ischemia. Stroke 16(3), 406-415 (1985).

14. Antiplatelet Trialists' Collaboration: Secondary prevention of vascular disease by prolonged antiplatelet treatment. Br. Med. J. (Clin. Res. Ed.) 296(6618), 320-331 (1988).

15. ESPS Group: European Stroke Prevention Study. Stroke 21(8), 1122-1130 (1990).

16. Diener HC, Cunha L, Forbes C, Sivenius J, Smets P, Lowenthal A: European Stroke Prevention Study 2. Dipyridamole and acetylsalicylic acid in the secondary prevention of stroke. J. Neurol. Sci. 143(12), 1-13 (1996).

17. Leonardi-Bee J, Bath PM, Bousser MG et al: Dipyridamole in Stroke Collaboration (DISC). Dipyridamole for preventing recurrent ischemic stroke and other vascular events: a meta-analysis of individual patient data from randomized controlled trials. Stroke 36(1), 162-168 (2005).

18. Sacco RL, Sivenius J, Diener H-C: Efficacy of aspirin plus extended-release dipyridamole in preventing recurrent stroke in high-risk populations. Arch. Neurol. 62, 403-408 (2005).

19. Theis JGW, Deichsel G, Marshall S: Rapid development of tolerance to dipyridamoleassociated headaches. Br. J. Clin. Pharmacol. 48, 750-755 (1999).

20. Lindgren A, Husted S, Staaf G, Ziegler B: Dipyridamole and headache - a pilot study of initial dose titration. J. Neurol. Sci. 223(2), 179-184 (2004).

21. Diener HC, Darius H, Bertrand-Hardy JM, Humphreys M: Cardiac safety in the European Stroke Prevention Study 2 (ESPS2). Int. J. Clin. Pract. 53(3), 162-163 (2001).

22. CAPRIE Steering Committee: A randomized, blinded, trial of clopidogrel versus aspirin in patients at risk of ischaemic events (CAPRIE). Lancet 348(9038), 13291339 (1996).

23. European Stroke Initiative Executive Committee and the EUSI Writing Committee: European stroke initiative recommendations for stroke management update 2003. Cerebrovasc. Dis. 16, 311-337 (2003).
24. Albers GW, Amarenco P, Easton JD, Sacco RL, Teal P: Antithrombotic and thrombolytic therapy for ischemic stroke. Chest 126(Suppl.), S483-S512 (2004).

25. Royal College of Physicians: National Clinical Guidelines for Stroke, 2nd Edition. Prepared by the Intercollegiate Stroke Working Party. Royal College of Physicians, London, UK (2004).

26. National Institute for Health and Clinical Excellence. Clopidogrel and modified-release dipyridamole in the prevention of occlusive vascular events - guidance. NICE, London, UK (2005).

www.nice.org.uk/pdf/TA090guidance.doc

27. Jones L, Griffin S, Palmer S et al: Clinical effectiveness and cost-effectiveness of clopidogrel and modified-release dipyridamole in the secondary prevention of occlusive vascular events: a systematic review and economic evaluation. Health Technol. Assess. 8(38), 1-196 (2004).

28. Topol EJ, Easton D, Harrington RA et al.: Blockade of the glycoprotein IIb/IIIa receptor to avoid vascular occlusion trial investigators. Randomized, double-blind, placebo-controlled, international trial of the oral IIb/IIIa antagonist lotrafiban in coronary and cerebrovascular disease. Circulation 108(4), 399-406 (2003). 